\title{
Augmented-Reality within Computer Assisted Orthopaedic Surgery Workflows: A Proof of Concept Study
}

\author{
Hisham Iqbal $^{1 *}$, Fabio Tatti $^{1 * *}$ and Ferdinando Rodriguez y Baena ${ }^{1 \dagger}$ \\ ${ }^{1}$ Mechatronics in Medicine Laboratory, Imperial College London, London, UK \\ hisham.iqbal13@imperial.ac.uk, f.rodriguez@imperial.ac.uk
}

\begin{abstract}
The integration of augmented-reality (AR) in medical robotics has been shown to reduce cognitive burden and improve information management in the typically cluttered environment of computer-assisted surgery. A key benefit of such systems is the ability to generate a composite view of medical-informatics and the real environment, streamlining the pathway for delivering patient-specific data. Consequently, AR was integrated within an orthopaedic setting by designing a system that captured and replicated the userinterface of a commercially available surgical robot onto a commercial head mounted see through display. Thus, a clinician could simultaneously view the operating-site and realtime informatics when carrying out an assisted patellofemoral-arthroplasty (PFA). The system was tested with 10 surgeons to examine its usability and impact on procedurecompletion times when conducting simulated PFA on sawbone models. A statistically insignificant mean increase in procedure completion-time $(+23.7 \mathrm{~s}, \mathrm{p}=0.240)$ was found, and the results of a post-operative qualitative-evaluation indicated a strongly positive consensus on the system, with a large majority of subjects agreeing the system provided value to the procedure without incurring noticeable physical discomfort. Overall, this study provides an encouraging insight into the high levels of engagement AR has with a clinical audience as well as its ability to enhance future generations of medical robotics.
\end{abstract}

\section{Introduction}

The research and development of novel visualisation techniques for medical informatics has recently led to increased integration of technologies such as augmented- and virtual-reality, AR and VR respectively, within the sphere of medical-robotics and computer-assisted surgical (CAS) procedures.

* Main Study Coordinator and drafted paper

** Experimental Coordinator

${ }^{\dagger}$ Principal Investigator 
In particular, interest in AR has led to studies investigating the use of head-mounted displays (HMDs) in surgical contexts with cited advantages including reduced visual scanning, faster detections of changes in patient vital-signs, and even lowered rates of physical burden experienced by clinicians [1].

A crucial benefit introduced by AR is the ability to provide real-time access to a composited view of visual-cues from multiple sources associated with CAS: surgical workflows, 2D/3D medical images, and the real-environment [2]. Delegating the task of rendering this information to a singular device has the potential to reduce the clutter and crowding issues typically associated with CAS, and to streamline the surgical experience.

To explore these potential benefits within an orthopaedic setting, an AR system-architecture was previously developed and benchmarked by the team of authors [3]; with this study exploring the expansion of the reported system with a view to providing a mixed-reality surgical workflow. The mixed-reality workflow was generated by capturing surgical-workflow images from a display unit on a commercially available surgical robot (NAVIO, Smith \& Nephew plc), and subsequently rendering this information onto a virtual heads-up display (HUD) displayed on a HoloLens (Microsoft, WA) HMD, a typical view of the user is included in Figure 1A.

This paper details a pilot study exploring the usability and impact on procedure completion time of the constructed mixed-reality workflow on simulated patellofemoral-arthroplasty (PFA) procedures using sawbones. The remainder of the paper is structured as follows; Section 2 details the systemarchitecture and the observed experimental-protocol. Section 3 reports experimental-results, and Section 4 concludes the paper with a discussion of results and concluding remarks.

\section{Materials and Methods}

\subsection{System Architecture}

To generate a mixed-reality surgical workflow, a capture card (AVerMedia GC553) was attached to the DisplayPort output of a surgical robot, and .jpeg workflow screenshots were captured onto a Windows 10 PC via a USB 3.0 connection. Captured frames were continuously transmitted via the universal datagram protocol (UDP) [4] to a client HoloLens-app via wireless communication, with a $\mathrm{C} \#$ software on the $\mathrm{PC}$ handling this data-transmission process. Packetised image data was decoded and rendered onto a HUD on the client-HoloLens end by a C\# app deployed via Unity (Unity Technologies, $\mathrm{SF}$ ). This resulted in an untethered surgical-workflow HUD, which could be manipulated with voicecommands and gestures (see Figure 1A). The system-testing protocol is detailed in Sections 2.2-2.4.

\subsection{Experimental Protocol}

Ten subjects were recruited over the course of the International Society for Technology in Arthroplasty (ISTA) conference between 10/10/18-13/10/18. Each subject carried out a standard and AR-enhanced patellofemoral arthroplasty (PFA), enabling a 'within-subject' study-design when evaluating of quantitative data. Each subject was randomly assigned an operating order (AR first/second) to prevent training-effect based tainting of the collected data. The measures collected during this study included operating time-cost and a post-operative qualitative evaluation. Prior to testing, subjects signed an informed consent form approved by the Imperial College Research Ethics Committee (ICREC ref: 18IC4769) which also approved the study as a whole.

\subsection{Measure 1: Operating Time-Cost}

The impact of AR on procedure-completion time was investigated by recording the elapsed procedural-time. Each subject had a pair of procedure-completion times: a standard and AR-enhanced 
robot-assisted PFA. These data were utilised to calculate a 'time-cost' corresponding to the additional amount of procedural-time associated with introducing the AR system.

\subsection{Measure 2: Qualitative Evaluation}

On trial completion, each of the 10 subjects completed a questionnaires (questions included in Figure 2) designed to evaluate the ergonomics and usability (physical comfort/distress, ease-of-use etc.) of the mixed-reality workflow. Survey responses were graded from 1-5 corresponding to the following options: strongly disagree, disagree, neutral, agree and strongly agree.

\section{Results}

Ten subjects $(9 \mathrm{M}, 1 \mathrm{~F})$ were recruited, with 4 subjects declaring prior CAS experience. Each subject carried out a standard and AR enhanced PFA procedure to completion.

\subsection{Measure 1: Operating Time-Cost Results}

The time cost was calculated for each subject $\left(T_{A R}-T_{\text {Standard }}\right)$, with a positive time-cost indicating a longer completion time with AR-enhancements. Figure 1B shows the time-costs generated by each subject, with the mean population time-cost calculated as $+23.7 \mathrm{~s}$. The time-cost dataset was verified to be normally distributed, following which a one sided paired t-test was carried out on the mean timecost value to examine if it was significantly larger than 0 , resulting in a p-value of 0.240 . This fell below the pre-set 5\% significance level indicating no statistically significant time-cost was associated with the use of the AR-workflow.

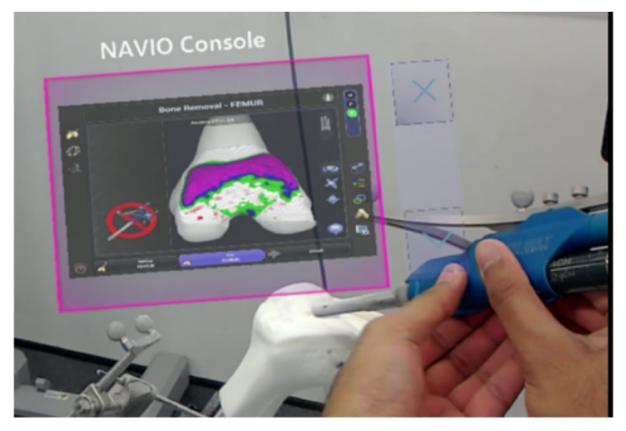

(A)

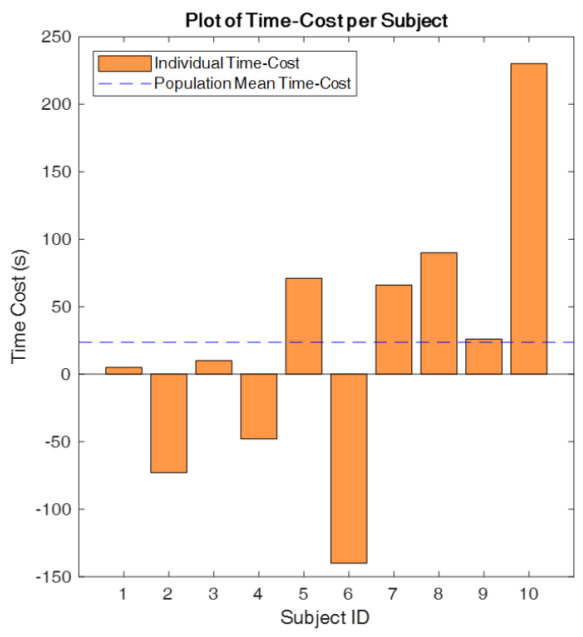

(B)

Figure 1 (A) - First person view of clinical user. A HUD is overlaid onto the real environment allowing a user to simultaneously view the operating site and surgical workflow information. (B) Bars indicate time-costs per subject and dashed line represents mean time-cost across population. 


\subsection{Measure 2: Qualitative Evaluation Results}

The Likert data from the survey was transcribed as described in Section 2.4, and then collated to create the plot seen in Figure 2. This figure illustrates the distribution of answers and mean Likert score for each survey question.

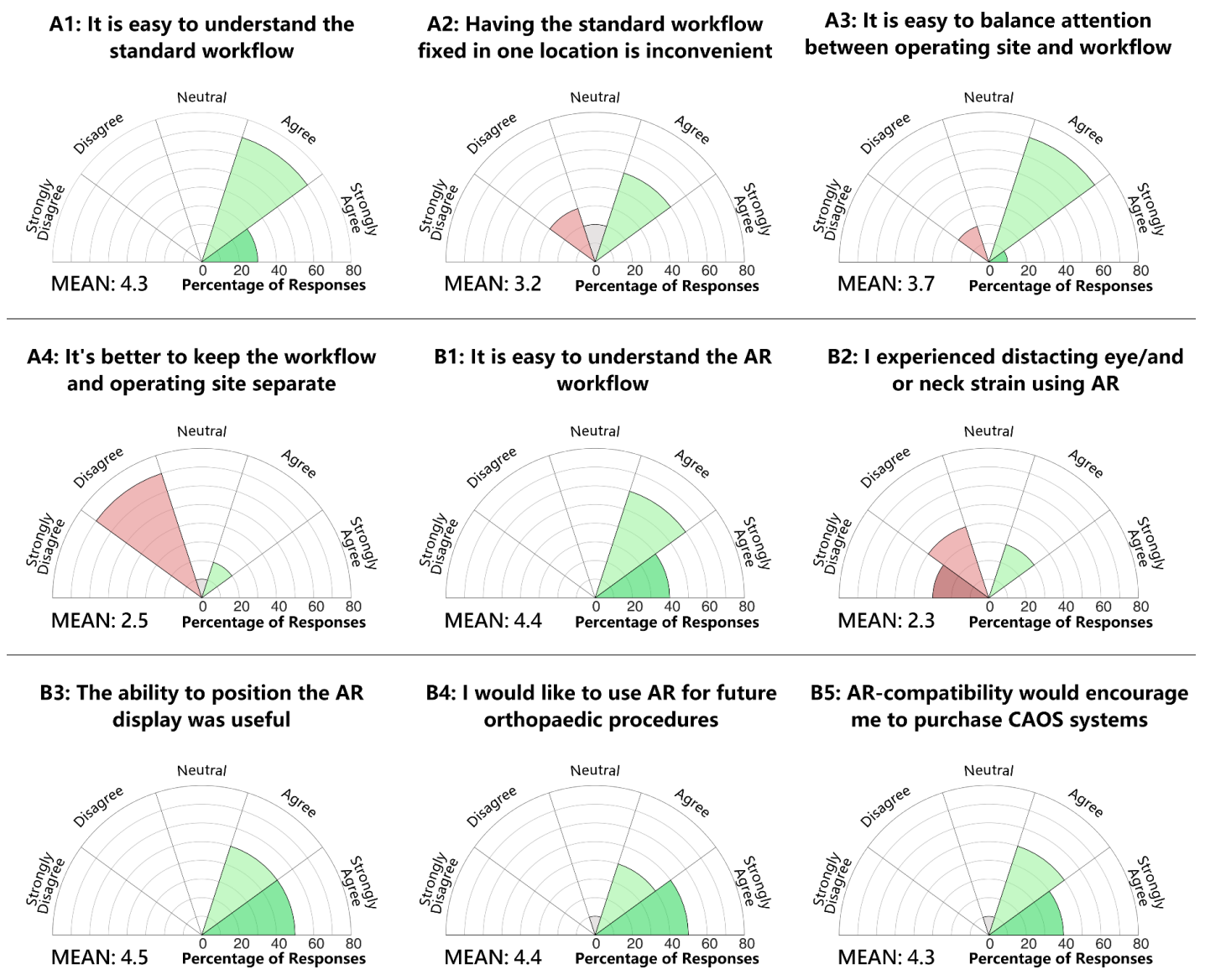

Figure 2 - Plot of questionnaire responses. Position of circular sector corresponds to answer, and radius of sector sized by proportion of responses. E.g. in A1, 70\% of respondents agree, and 30\% strongly agree. Mean Likert-score has also been included to contextualise 'average' response.

\section{Discussion}

As highlighted within Section 3.1, the mean time-cost due to AR was 23.7s. Whilst this figure is skewed by one clear outlier exhibiting a strong training-effect, the results remain statistically insignificant $(p=0.24, \alpha=0.05)$. This indicated that the use of AR was not associated with a significant increase in procedure-completion times.

Furthermore, it can be reasonably hypothesised that this time-cost will decrease with repeated exposure to AR, as indicated by a range of studies in literature that indicate AR provides parallel [5], or shorter learning curves [6] in the execution and training of manual tasks. 
In addition, the findings of the qualitative evaluation present results indicating this study population had positive opinions on the AR workflow. In particular, the functionality of the system appears validated with users strongly agreeing on the system adding value to the procedure (B3, B4). The system did not appear to compromise any physical comfort (B2), and appears to have engaged the study population who agreed on average they would return to using similar technology in the future (B5). These findings are consistent with previous studies, which have found high levels of engagement and user satisfaction when utilising AR within assisted-surgical tasks [7, 8].

Overall, the findings of this study present an encouraging indication that AR has the potential to add value to CAOS-procedures without significantly impairing operating times alongside a high level of engagement, providing a picture of the willingness of clinicians to adopt AR technologies in the next generation of medical robotics and surgery.

\section{Disclosures}

This project has been kindly supported by the EPSRC and TJ Smith \& Nephew Ltd. in the form of an industrial CASE Studentship. The study also received support from Innovate UK under the project number 103950.

\section{References}

[1] Yoon, J., Chen, R., Kim, E., Akinduro, O., Kerezoudis, P., Han, et al.(2018). Augmented reality for the surgeon: Systematic review. The International Journal of Medical Robotics and Computer Assisted Surgery, 14(4), p.e1914.

[2] Vávra, P., Roman, J., Zonča, P., Ihnát, P., Němec, et al. (2017). Recent Development of Augmented Reality in Surgery: A Review. Journal of Healthcare Engineering, 2017, pp.1-9.

[3] Iqbal, H. and Rodriguez Y Baena, F. (2018). Benchmarking of a Proposed Augmented-Reality System Architecture. Beijing: EPiC Series in Health Sciences, pp.87-93.

[4] Huh, J. (2018). Reliable User Datagram Protocol as a Solution to Latencies in Network Games. Electronics, 7(11), p.295.

[5] Hou, L. and Wang, X. (2013). A study on the benefits of augmented reality in retaining working memory in assembly tasks: A focus on differences in gender. Automation in Construction, 32, pp.3845.

[6] Vera, A., Russo, M., Mohsin, A. and Tsuda, S. (2014). Augmented reality telementoring (ART) platform: a randomized controlled trial to assess the efficacy of a new surgical education technology. Surgical Endoscopy, 28(12), pp.3467-3472.

[7] Condino, S., Turini, G., Parchi, P., Viglialoro, et al. (2018). How to Build a Patient-Specific Hybrid Simulator for Orthopaedic Open Surgery: Benefits and Limits of Mixed-Reality Using the Microsoft HoloLens. Journal of Healthcare Engineering, 2018, pp.1-12.

[8] Botden, S., de Hingh, I. and Jakimowicz, J. (2008). Suturing training in Augmented Reality: gaining proficiency in suturing skills faster. Surgical Endoscopy, 23(9), pp.2131-2137.

The layout settings of this guide are set to conform to the requirements of both the EPiC Series and Kalpa Publications. If the settings are altered, the requirements below need to be kept in mind, since papers deviating from the formatting standards will not be accepted for printing. This section is 